\title{
Comparison of Compression Algorithms' Impact on Fingerprint and Face Recognition Accuracy
}

\author{
A. Mascher-Kampfer ${ }^{1, *}$ Herbert Stögner ${ }^{1}$, and Andreas Uhl ${ }^{1,2}$ \\ ${ }^{1}$ School of Telematics \& Network Engineering, Carinthia Tech Institute (CTI), Austria \\ ${ }^{2}$ Department of Computer Sciences, Salzburg University, Austria
}

\begin{abstract}
The impact of using different lossy compression algorithms on the matching accuracy of fingerprint and face recognition systems is investigated. In particular, we relate rate-distortion performance as measured in PSNR to the matching scores as obtained by the recognition systems. JPEG2000 and SPIHT are correctly predicted by PSNR to be the most suited compression algorithms to be used in fingerprint and face recognition systems. Fractal compression is identified to be least suited for the use in the investigated recognition systems, although PSNR suggests JPEG to deliver worse recognition results in the case of face imagery. JPEG compression performs surprisingly well at high bitrates in face recognition systems, given the low PSNR performance observed.
\end{abstract}

Keywords: fingerprint recognition, face recognition, image compression, biometrics

\section{INTRODUCTION}

With the increasing usage of biometric systems the question arises naturally how to store and handle the acquired sensor data. In this context, the compression of these data may become imperative under certain circumstances due to the large amounts of data involved. Among other possibilities (e.g. like template storage on IC cards), compression technology may be used in two stages of the processing chain in classical biometric recognition:

1. Storage of reference data: In most template databases (where the reference data of the enrolled individuals is stored) only the extracted features required for the matching step are stored as opposed to retaining the originally acquired sensor data. However, in case the features should be replaced for some reason (e.g. when a superior or licence-free matching technique involving a different feature set becomes available), having stored only extracted features implies the requirement for all legitimate users for a reenrollment, which can be expensive and is highly undesired since user-acceptance of the entire biometric system will suffer. Storing the original sensor data in addition to the features required for the current matching technique solves this problem. Of course, these data need to be stored in compressed (to save storage space) and encrypted (to protect privacy) form.

2. Transmission after sensor data acquisition: In distributed biometric systems, the data acquisition stage is often dislocated from the feature extraction and matching stage (this is true for the enrollment phase as well as for authentication). In such environments the sensor data have to be transferred via a network link to the respective location, often over wireless channels with low bandwidth and high latency. Therefore, a minimization of the amount of data to be transferred is highly desirable, which is achieved by compressing the data before transmission. An alternative solution would be to extract the features before transmission and to transfer feature data only - in many cases, feature extraction is more demanding as compared to compression which generates additional workload for the often mobile and low power acquisition devices.

* This artificial name represents a group of students working on this paper at CTI in the framework of a system security lab (summer term 2006): Michael Dorfer, Severin Kampl, Alexander Maier, Daniel Mark, Andreas Palli, and Günter Scheer.

E-mail: uhl@cosy.sbg.ac.at 
Having found that compression of the raw sensor data can be advantageous in certain applications, we have to identify techniques suited to accomplish this task in an optimal manner. In order to maximize the benefit in terms of data reduction, lossy compression techniques have to be applied. However, the distortions introduced by compression artifacts may interfere with subsequent feature extraction and may degrade the matching results. In particular, FRR or FNMR will increase (since features of the data of legitimate users are extracted less accurately from compressed data) which in turn affects user convenience and general acceptance of the biometric system. In extreme cases, even FAR or FMR might be affected.

In this work, we will focus on the compression of fingerprint and face images. Contrasting to the overwhelming majority of literature and studies in this field, we will not rely on assessing the resulting objective and subjective image quality after compression only, but we will apply biometric recognition systems to the compressed sensor data to evaluate the effects of compression on recognition accuracy, in particular on the matching results of legitimate users.

In Section 2, we will review and discuss the available literature on fingerprint and face image compression. Section 3 is the main part of this work where we first describe the employed image compression techniques, the used biometric recognition systems, and the data these algorithms are applied to. Subsequently we present and discuss our experimental results where we compare and rank the compression algorithms in use. Section 4 concludes the paper.

\section{FINGERPRINT AND FACE IMAGE COMPRESSION}

The reason for focussing our investigations to fingerprint and face recognition is that these two biometric modalities are expected to be used in EU travel documents - the ePassport. In this application scenario, compression technology is even more interesting as compared to classical biometrical recognition applications due to the required storage of template data on IC cards.

During the last decade, several algorithms and standards for compressing image data relevant in biometric systems have evolved. The certainly most relevant one is the recent ISO/IEC 19794 standard on Biometric Data Interchange Formats, where Parts 4 and 5 cover fingerprint and face image data, respectively. ISO/IEC 19794-4 allows fingerprint image data to be stored in lossy manner in the JPEG, ${ }^{1}$ WSQ, ${ }^{2}$ and JPEG2000 ${ }^{3}$ formats, where the latter is recommended. A corresponding specific JPEG2000 Part I profile for 1000 ppi fingerprint images is proposed in. ${ }^{4}$ ISO/IEC 19794-5 allows face image data to be stored in lossy manner in the JPEG and JPEG2000 formats.

While the data formats specified by the ISO/IEC 19794 standard are fixed at present state, their impact on recognition accuracy as compared to other algorithms is not well documented. Moreover, many more generalpurpose algorithms as well as techniques tailored to specific biometric modalities exist, which we will discuss in the following.

A vast amount of literature exists in which different compression algorithms are compared with respect to their rate-distortion performance on "usual" image data (e.g. ${ }^{5,6}$ ). Also, more specific image data has been used for comparisons: for example, ${ }^{7}$ compares wavelet transforms and fractal coding in texture-based image retrieval, in earlier work we have used a prediction (DPCM) scenario $^{8}$ and artificially generated images ${ }^{9}$ for assessing various algorithms.

Fingerprint images exhibit characteristic high energy in certain high frequency bands resulting from the ridge-valley pattern and other structures. To account for this property, the WSQ standard for lossy fingerprint compression as adopted by the $\mathrm{FBI}^{2}$ uses a specific wavelet packet subband structure which emphasises the important high frequency bands. This standard has been shown to be clearly superior to JPEG compression in terms of psychovisual and PSNR quality. ${ }^{10}$

Wavelet-based compression schemes have been subsequently investigated in great number for their usefulness in fingerprint compression. Filter choice in classical pyramidal coding schemes specifically tuned for fingerprint compression has been one of the main areas of research. ${ }^{11,12}$ identify biorthogonal filters as being superior to orthogonal ones. ${ }^{13}$ also optimizes filters for fingerprint compression, a special type of wavelet filter (polyphase) to adapt to high frequency content is suggested in. ${ }^{14}$ Simulated annealing over the wavelet filter coefficients ${ }^{15}$ 
is used as well as a method based on the coevolutionary genetic algorithm ${ }^{16}$ to evolve specialized wavelets for fingerprint images (the latter work with focus on the JPEG2000 context).

Inspired by the WSQ FBI algorithm, a few wavelet packet based fingerprint compression schemes have been developed. An improved WSQ entropy coding stage using lossless zerotree coding is proposed in. ${ }^{17}$ Various subband structures with subsequent lattice vector quantization as proposed in ${ }^{18}$ turn out to be superior to JPEG, WSQ, EZW, and SPIHT among others. ${ }^{19}$ also uses the concept of adaptive subband structures to improve on the fixed structure WSQ method, ${ }^{20}$ adapts the wavelet packet filters and the decomposition level in addition to the selection of subbands and adapts better to the actual frequency characteristics of the image in this way.

A final class of compression schemes exploits the strong directional features in fingerprint images caused by ridges and valleys. A scanning procedure following dominant ridge direction has shown to improve lossless coding results as compared to JPEG-LS and PNG. ${ }^{21}$ A wavelet footprint representation characterizing efficiently singular structures (corresponding to ridges) is used $\mathrm{in}^{22}$ which turns out to deliver better results as compared to the SPIHT algorithm. ${ }^{23}$ Contourlets $^{24,25}$ and contourlet packets ${ }^{26}$ are used to exploit directional information which also results in improvements as compared to classical algorithms.

It is worth noticing that the evaluation of compression performance of the schemes described so far is surprisingly limited to PSNR or RMS-error computation and to psychovisual studies. Effects on actual fingerprint matching or recognition are not investigated. One of the few exceptions is found in $^{27}$ where image tiles containing minutiae are compressed using PC and wavelet transform and the effects on FAR of a specific recognition system are studied. However, this is a very specialized technique since only parts of the original sensor data containing minutiae are retained.

Contrasting to fingerprints, face images represent typical average image data with dominating low and medium frequency content. Therefore, apart from ${ }^{28}$ (which reconstructs facial texture images with partial areas of reference face pictures by least-square minimization), no specific compression techniques have been specified and investigated for face images, for most applications simply JPEG or JPEG2000 compression is used. Contrasting to the fingerprint case, compression effects with respect to face recognition accuracy have been studied: ${ }^{29}$ investigates the impact of JPEG und JPEG2000 on the recognition results of 12 different face recognition techniques, and ${ }^{30}$ employs varying bitrates using JPEG2000 to determine the trade-off points between recognition accuracy and compression ratio.

In the subsequent experimental study we will apply a set of general purpose compression algorithms to fingerprint and face image data and we will study their respective impact on the recognition accuracy of selected matching techniques. Specifically, we will relate the rate-distortion performance of the compression schemes to the matching score of the recognition systems applied to the compressed data. In this way, we are able to compare the compression schemes for their respective usefulness in the biometric context and we will also see whether we obtain similar or different results for the two investigated biometric modalities.

\section{EXPERIMENTAL STUDY}

\subsection{Setting and Methods}

\subsubsection{Compression Algorithms}

We use five different general purpose image compression algorithms which have been extensively described and discussed in the open literature before (implementations are available for free download on the internet). Since the block-DCT based JPEG standard $^{1}$ is a part of almost any image processing tool we do not give further reference on it. For the wavelet-based JPEG $2000^{3}$ standard we have used the JAVA reference implementation $\mathrm{JJ}_{2000}{ }^{\dagger}$. SPIHT ${ }^{23}$ relies as well on the wavelet transform, but contrasting to JPEG2000 which exploits intra subband correlations only, SPIHT is a zero-tree based codec which exploits coefficient dependencies across subbands. We have employed the implementation as provided by the developers originally ${ }^{\ddagger}$. In addition to these described three transform-based compression algorithms we also use codebook-based schemes. Fractal image compression ${ }^{31}$ exploits self similarities within images (where the image itself may be interpreted as an

\footnotetext{
${ }^{\dagger}$ http://jj2000.epfl.ch/

${ }^{\ddagger}$ http://www.cipr.rpi.edu/research/SPIHT/
} 
internal codebook) and has been discussed controversially in the literature. We have employed an adaptive quadtree method implementation ${ }^{\S}$ based on Y. Fishers code. As the fifth algorithm a special flavour of vector quantization is used. PRVQ performs a block-based prediction within the image to be compressed (similar to the intra-prediction mode in H.264), subsequently the residual image is compressed by a vector quantization strategy. ${ }^{9}$ describes PRVQ and the used fractal compression variant in some detail.

When applied to common images, JPEG2000 and SPIHT usually give the best results in terms of PSNR, closely followed by PRVQ. For high bitrates, JPEG provides fourth best results, whereas fractal compression is superior to JPEG for medium and low bitrates. We expect similar results for face images, whereas for fingerprint images it may be expected that the three block-based schemes (JPEG, fractal compression, and PRVQ) might not be able to preserve the high frequency ridge structure well.

\subsubsection{Biometric Recognition Systems}

The employed recognition systems have been selected to ensure reproducibility of the results - corresponding software is available for free download on the internet.

Fingerprint Recognition We employ three fingerprint systems. VeriFinger\| is a commercial tool which relies on minutiae matching without employing core and delta points and claims tolerance to translation and rotation. The algorithm outputs a similarity score ranging between 700-1200 for identical images and 0 for entirely different fingerprints (this is the actual mean value of the scores between our first image and the five remaining fingerprints). eFinger** is a students' project work using the minutiae matching paradigm as well. The classical stages enhancement and ridge detection, binarization, thinning, minutiae extraction, and matching are employed. The algorithm outputs as well a similarity measure in the "Image Mapping" mode ranging between 300-500 for identical images and 62 for different fingerprints (which is again the mean value as described above). Fingerprint Recognition System ${ }^{\dagger \dagger}$ (FiRS) is a commercial Matlab tool using Gabor filters to capture both local and global details in a fingerprint as a compact fixed length FingerCode (similar to the well known IrisCode). Matching computes Euclidian distance between two FingerCodes and outputs a value in the range between 0 for identical images and a mean value of 2014 for different fingerprints.
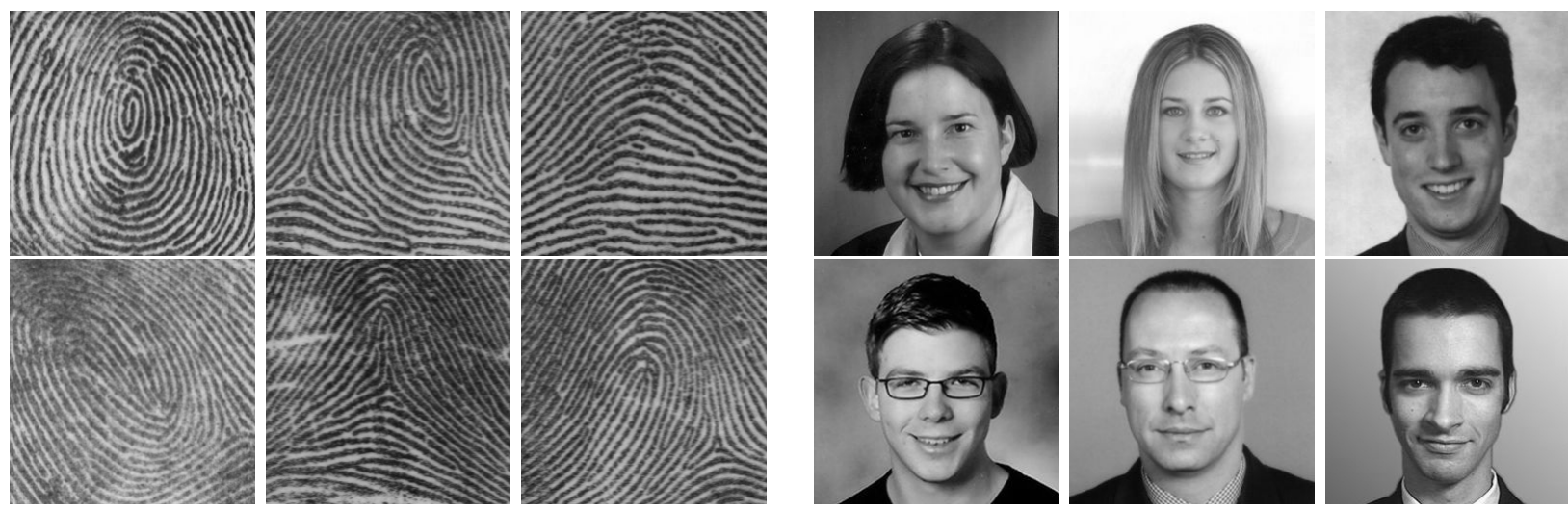

Figure 1. Fingerprint and frontal Face images used in experiments.

\footnotetext{
${ }^{\S}$ http://www . verrando.com/ verrando/pulcini/gp-ifs1.html

ॠhttp://www.ganesh.org/webcomp/images/

"http://neurotechnologija.com

${ }^{* *}$ http://sourceforge.net/projects/efinger

${ }^{\dagger \dagger}$ http://utenti.lycos.it/matlab/beginner.htm
} 
Face Recognition We employ two face systems. VeriLook* is a tool developed by the company commercializing VeriFinger. Although there is no description of the algorithm available we conjecture that a correlation type measure after image registration is used which outputs a similarity measure in the range between 1.0 for identical images and 0.32 (again the described mean value) for different faces. Note also that the maximal similarity encountered among our test images is 0.47. Face Recognition System ${ }^{\dagger}$ (FaRS) is a commercial Matlab tool using eigenfaces as significant features for matching. The algorithm computes a distance measure and outputs a value of 0 for identical images and a mean value of 169577500 for different faces.

\subsubsection{Sample Data}

For both biometric modalities we consider $256 \times 256$ pixels images with 8 bit grayscale information per pixel (8bpp). All employed compression software is able to handle this type of imagery (which is not true for color or non-squared images). The six fingerprint images used in the following experiments as shown in Fig. 1 have been extracted from a sample fingerprint database ${ }^{\ddagger}$.

The six frontal face images used in the experiments as shown in Fig. 1 have been extracted from several databases - exact reference can be provided upon request.

\subsection{Results}

In the concrete experiments we compress a fingerprint or face image with a series of certain compression ratios and record both ratio and corresponding PSNR values. Subsequently, we conduct a fingerprint or face recognition procedure, matching the compressed template against the uncompressed original image data. For example, the resulting score corresponds to a recognition situation where the enrollment samples have been stored in original form, whereas the acquired sensor data in the authentication stage has been transmitted to the matching unit in compressed form. In case of severe distortions, a poor matching result might cause a false rejection or false non-match.

\subsubsection{Fingerprint Images}

Fig. 2(a) displays the rate-distortion performance (compression ratio vs. PSNR) of JPEG applied to the fingerprint images in our test set. We notice differences up to $3 \mathrm{~dB}$ among the images, which is surprisingly high given the high structural similarity. Also the achievable compression ratio varies significantly with the lowest quality setting: ratios between 30 and 45 are obtained.

Fig. 2(b) shows the matching results of the VeriFinger system (compression ratio vs. similarity). An interesting observation is that we do not see monotonically decreasing similarity scores for increasing compression ratio (this is especially pronounced for img1-3). Except for fractal coding, this undesired property of VeriFinger is found when used combined with all compression techniques (though less pronounced for JPEG2000 and SPIHT). It is worth noting that the eFinger matching procedure does not exhibit the same effects although it is based on minutiae matching as well.

In Fig. 3(a), we display the rate-distortion performance (compression ratio vs. PSNR) of JPEG2000. Compared to the JPEG results (see Fig. 2(a)) PSNR values are consistently higher: for ratio 10, PSNR ranges between $31.5 \mathrm{~dB}$ and $34.5 \mathrm{~dB}$ for JPEG2000 and between $30 \mathrm{~dB}$ and $32 \mathrm{~dB}$ for JPEG, respectively; for ratio 30, PSNR is down to a range between 20dB and 23.5dB for JPEG, whereas JPEG2000 still delivers values between $24.5 \mathrm{~dB}$ and $27.5 \mathrm{~dB}$. However, the ranking among the images is identical for JPEG and JPEG2000.

The similarity scores of the eFinger system under JPEG2000 compression are visualized in Fig. 3(b). Contrasting to the VeriFinger results (compare Fig. 2(b)), increasing compression ratio causes monotonically decreasing scores.

However, we observe another interesting phenomenon. The scores corresponding to img 1 indicate the highest similarity degree among all images. Interestingly, img1 exhibits the lowest PSNR values of all images considered (see Fig. 3(a)). The opposite would have been expected of course - does this imply that PSNR is an extremely

\footnotetext{
*http://neurotechnologija.com

${ }^{\dagger}$ http: //www. advancedsourcecode.com/face. asp

${ }^{\ddagger}$ http://www . biometrix.at/fp-images.zip
} 


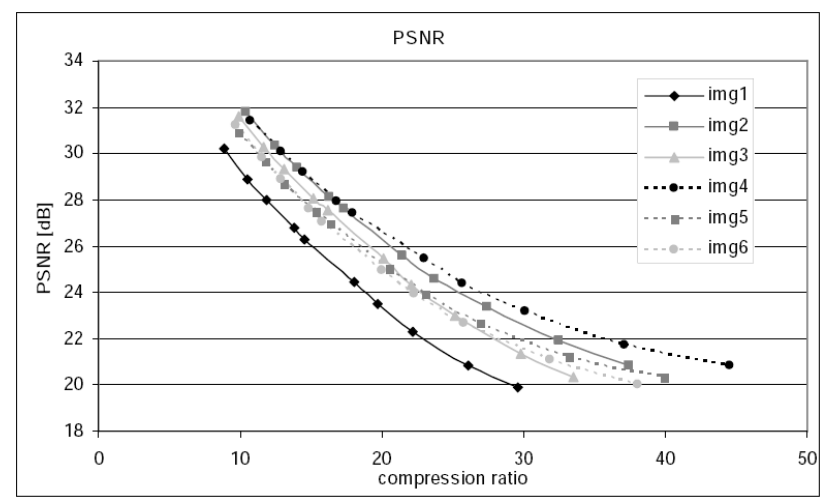

(a) rate-distortion performance

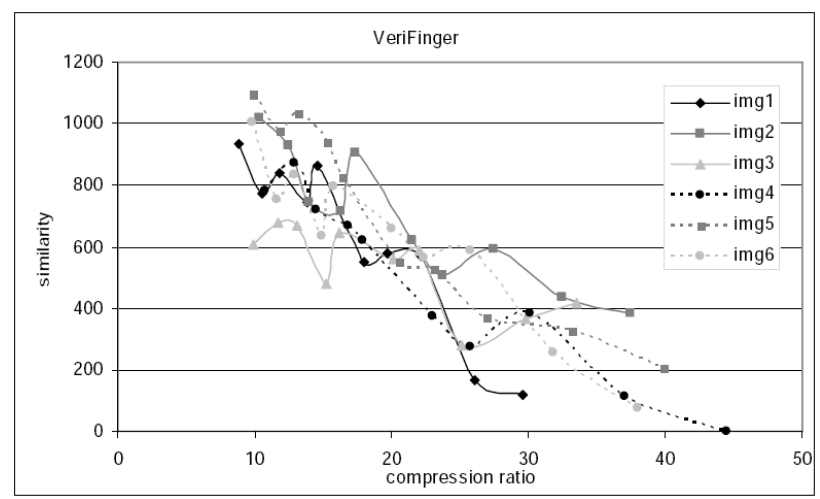

(b) VeriFinger matching scores

Figure 2. JPEG compression on fingerprint images.

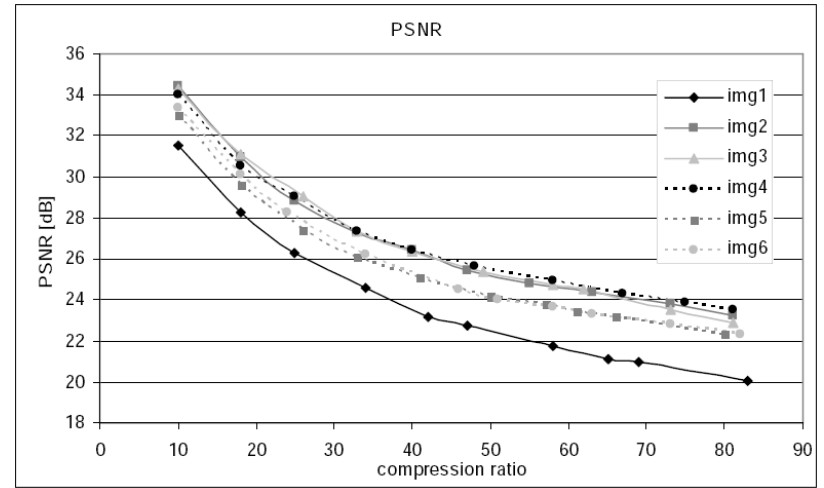

(a) rate-distortion performance

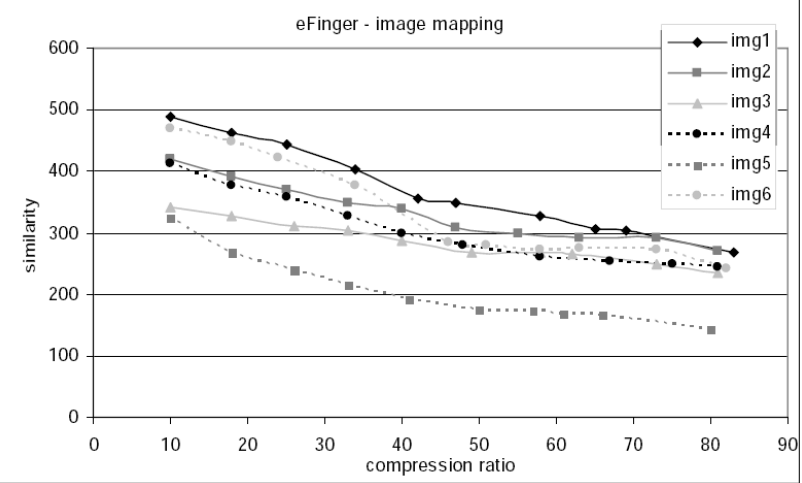

(b) eFinger matching scores

Figure 3. JPEG2000 compression on fingerprint images.

poor indicator of matching scores ? Not yet - the actual similarity value computed by the eFinger matching module is more dependent on the image structure (e.g. the number of minutiae) than on image quality. This gets clear when considering the similarity scores for img3. This image is among the Top 3 concerning PSNR across the entire range of bitrates but shares the lowest similarity value with img1 at a compression ratio of 10 . With increasing compression ratio, the similarity scores are much less deteriorated as compared to other images. The less steep slope of the similarity curve of img3 is the result of the better PSNR values.

In the following we present results averaged across all images considered. Fig. 4(a) compares the ratedistortion performance (compression ratio vs. PSNR) of all five compression algorithms. It is clearly visible that JPEG2000 and SPIHT behave very similarly and significantly outperform the remaining techniques across the entire range of bitrates. JPEG and PRVQ perform almost equivalently up to a compression ratio of 20 , for lower bitrates PRVQ is clearly superior. Fractal compression delivers very poor results for all bitrates. It should be noted that the absolute PSNR values are rather low which confirms that fingerprint images are not easy to compress, additionally we observe fairly large differences among the algorithms. JPEG and PRVQ have been used with their respective lowest quality settings resulting in a top compression ratio of about 40 . 


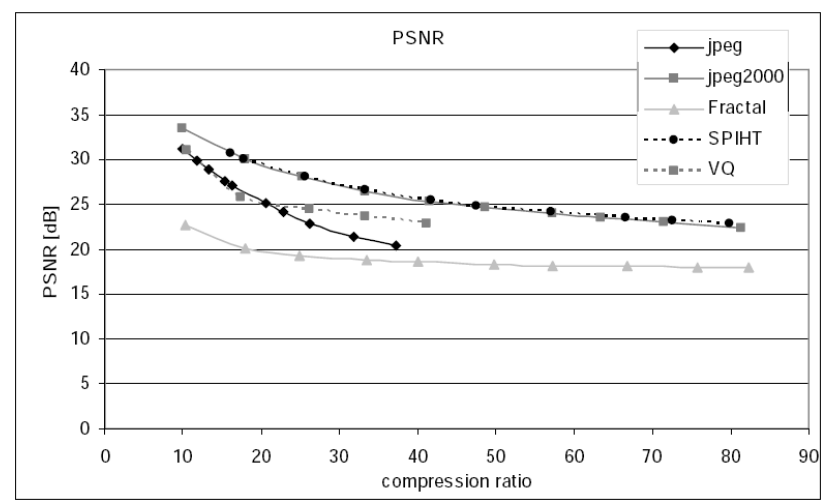

(a) rate-distortion performance

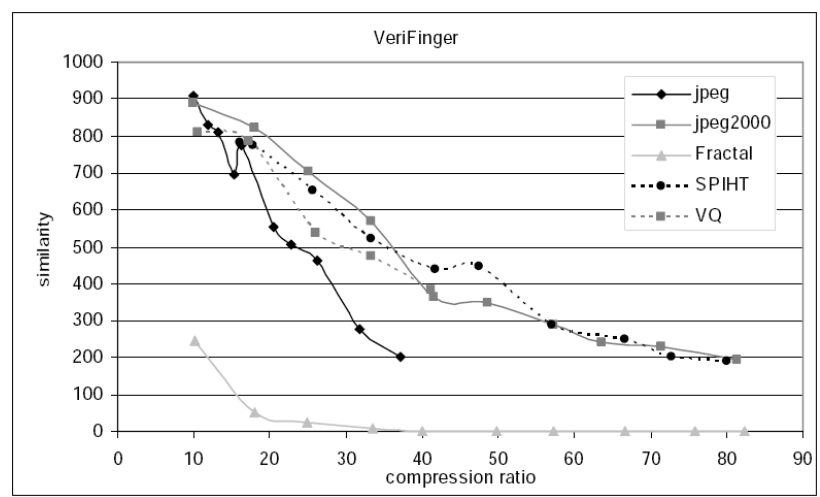

(b) VeriFinger matching scores

Figure 4. Averaged behaviour of compression algorithms applied to fingerprint images.

Subsequently, we will investigate whether the relative and absolute PSNR performance of the compression algorithms is a good indicator for the matching performance of the recognition systems when applied to the compressed data. Fig. 4(b) shows the averaged (over all images) results for VeriFinger. Surprisingly, JPEG delivers an excellent similarity score for compression ratio 10 (i.e. high quality) - in this bitrange range, only JPEG2000 shows competitive results. When bitrate is decreased, the similarity score of JPEG degrades significantly. PRVQ gives clearly better similarity values as compared to JPEG and at ratio 40 the PRVQ score is even identical to that of JPEG2000. Overall, JPEG2000 and SPIHT clearly outperform the rest of the compression algorithms. At the lower end, fractal compression exhibits the lowest similarity scores and attains a score of 0 for compression ratios 35 and higher. Recall that also entirely different images result in a similarity score of 0.

Fig. 5(a) presents the corresponding results for the eFinger system, which give a much more regular impression as compared to those of VeriFinger, although both systems rely on similar technology. Similarity scores of JPEG2000, SPIHT, and PRVQ correspond well to their respective PSNR results as shown in Fig. 4(a). However, JPEG shows consistently lower similarity scores as compared to PRVQ, contrasting to PSNR performance which is equal up to ratio 20 for both algorithms. Fractal compression again delivers the lowest similarity scores and attains values lower than 62 for ratios of 40 and higher (recall that 62 is the mean similarity value among our test images).

Finally, Fig. 5(b) shows the results of the FiRS. We observe the same ranking among the compression algorithms as observed with the eFinger system. Also the JPEG distance values are only competitive at ratio 10, for higher ratios PRVQ and the other algorithms are clearly better. Again fractal compression performs significantly weaker as the other algorithms and the distance score rises above the mean value among different images (i.e. 2014) for compression ratios of 25 and higher.

Overall, PSNR is found to be a satisfying but not perfect indicator for matching performance of the fingerprint recognition systems considered. In particular, the PSNR top-ranked algorithms JPEG2000 and SPIHT exhibit top-ranked recognition scores as well. Also, fractal compression confirms low recognition scores as expected due to the low PSNR results. However, the actual recognition scores of fractal compression are even lower as would have been expected and values identical to those obtained by entirely different images are seen. Fractal compression is obviously not at all suited for fingerprint compression and might actually lead to an increasing FAR/FMR in addition to increasing FRR/FNMR when used in fingerprint recognition. PRVQ does surprisingly well and outperforms JPEG almost across the entire range of compression ratios which is not expected given the PSNR performance only. JPEG on the other hand gives better recognition scores for compression ratio 10 as to be expected from PSNR results, the recognition scores for higher compression ratios, especially when compared to those of PRVQ are weaker as would have been expected. 


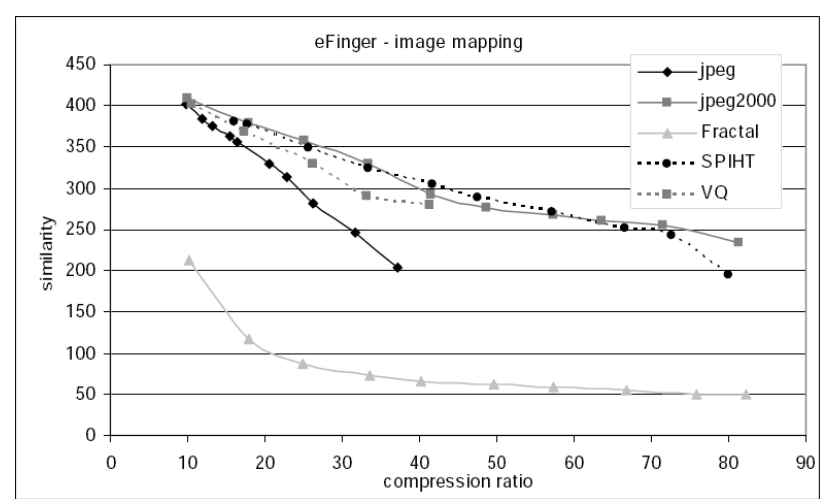

(a) eFinger matching score

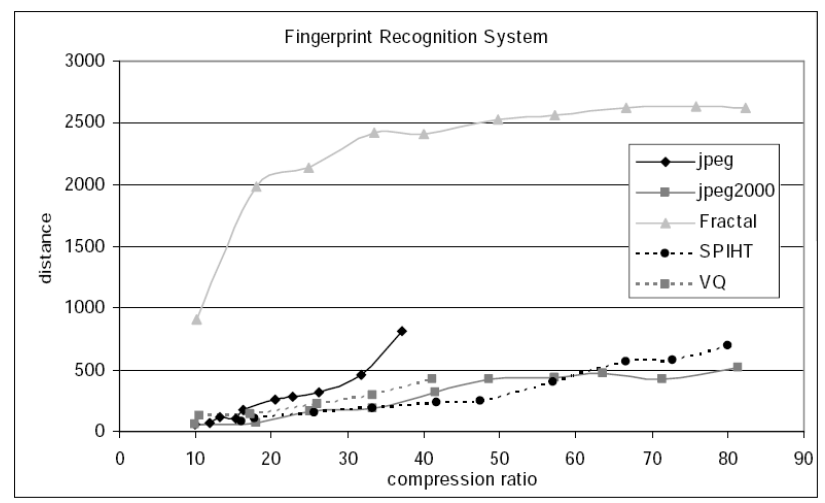

(b) Fingerprint Recognition System matching scores

Figure 5. Averaged behaviour of compression algorithms applied to fingerprint images.

\subsubsection{Face Images}

Fig. 6(a) displays the rate-distortion performance (compression ratio vs. PSNR) of SPIHT applied to the six face images. We notice differences up to $3 \mathrm{~dB}$ among the different images.

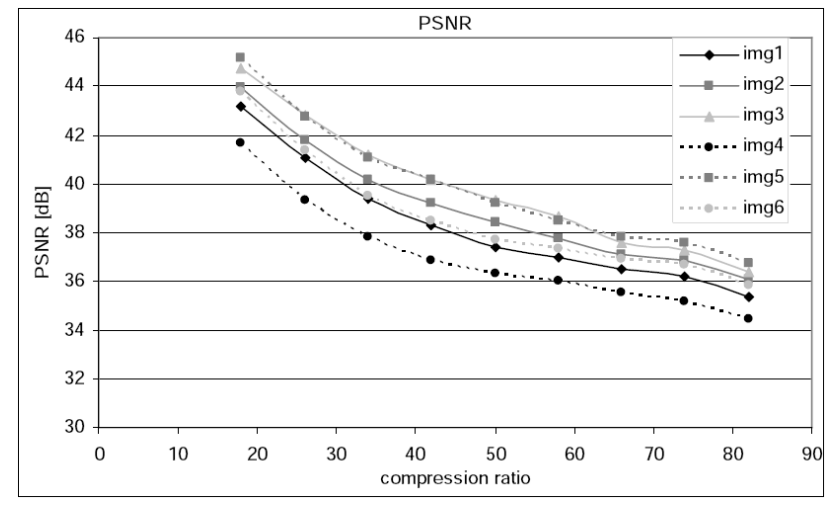

(a) rate-distortion performance

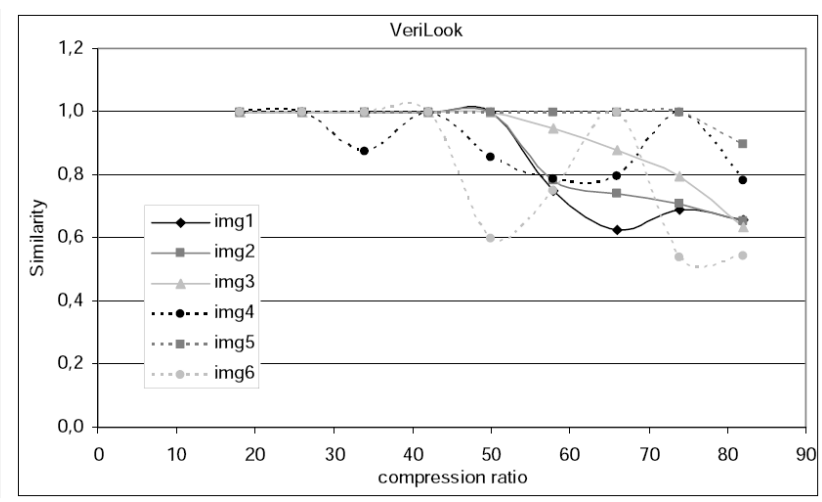

(b) VeriLook Matching scores

Figure 6. SPIHT compression on face images.

In Fig. 6(b) we present the matching results of the VeriLook system (compression ratio vs. similarity). Similar to the VeriFinger system provided by the same manufacturer (compare Fig. 2(b)) we do not see monotonically decreasing similarity scores for increasing compression ratio (this is especially pronounced for img1, img4, and img6). This undesired property of VeriLook is found when used in combination with all compression techniques investigated. It is worth noting that the FaRS matching procedure does not exhibit such effects.

In accordance to the fingerprint image case, we present in the following results averaged across all face images considered. Fig. 7(a) compares the rate-distortion performance (compression ratio vs. PSNR) of all five compression algorithms investigated. In general the PSNR values observed are significantly higher as compared to the fingerprint case. This is due to the already mentioned high frequency nature of fingerprint images, which cannot be modeled well with general purpose image compression schemes. 
Contrasting to the fingerprint case, SPIHT outperforms JPEG2000 consistently by 0.5dB. PRVQ does fairly well and is ranked third, even approaching the results of JPEG2000 for higher compression ratios. Fractal compression is clearly ranked at the forth position except for compression ratio 10 where JPEG is superior. JPEG performs worst and is the only algorithm not able to reach the maximal compression ratio 80.

We notice a significantly different result as compared to fingerprint images, especially at the lower end. Whereas fractal compression is the weakest compression scheme for fingerprint images, JPEG gives the worst PSNR values for face images. PRVQ is much improved for face images as compared to fingerprint images and even approaches JPEG2000 results for high compression ratios. Note that the ranking as seen for face images corresponds to the ranking as observed for common images as reported in literature.

It will be interesting to see in how far this different rate-distortion performance for face images is propagated into the recognition scores.

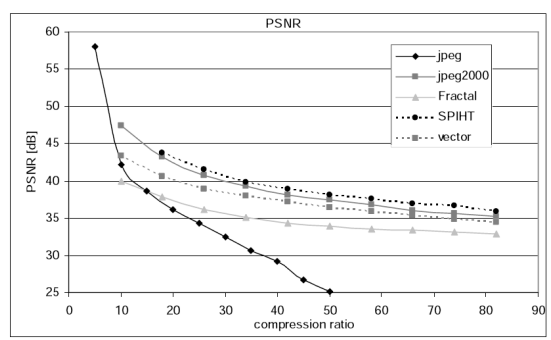

(a) rate-distortion performance

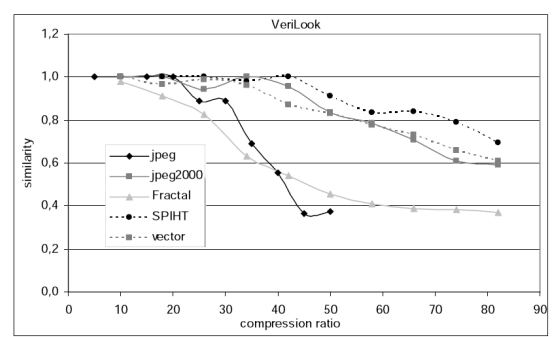

(b) VeriLook matching scores

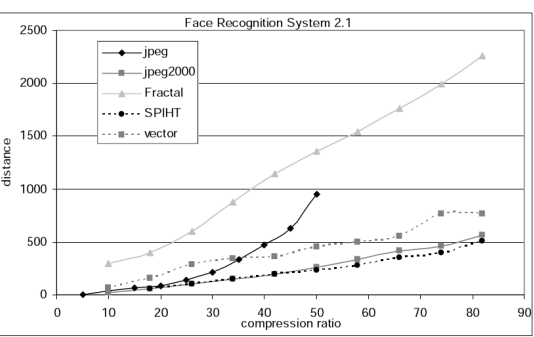

(c) Face Recognition System matching scores

Figure 7. Averaged behaviour of compression algorithms applied to face images.

Subsequently, we will investigate whether the relative and absolute PSNR performance of the compression algorithms is a good indicator for the matching performance of the face recognition systems. Fig. 7(b) shows the averaged (over all images) results for VeriLook. We notice two interesting issues. First, the similarity scores for JPEG are above those of fractal compression up to a compression ratio of 40 (although the PSNR values are significantly below those of fractal compression), JPEG even surpasses PRVQ up to a ratio of 20 in terms of similarity results. Second, PRVQ similarity scores for high compression ratios are slightly above those of JPEG2000 - but considering PSNR JPEG2000 is clearly superior. It is also worth noting that the maximal similarity score for different images in our test set was found to be 0.47 - the values for JPEG as well as for fractal compression fall below this threshold for compression ratios higher than 45 . In order to bound FAR/FMR and FRR/FNMR those two compression algorithms should never be used within a VeriLook application.

Finally, Fig. 7(c) shows the averaged results of the FaRS. Whereas the ranking in terms of distance scores corresponds well to the PSNR results for JPEG2000, SPIHT, and PRVQ, fractal compression results in the highest distance values of all algorithms although it was clearly ranked fourth in terms of PSNR. Again, JPEG does exceptionally well in terms of distance score as compared to its PSNR performance: up to a compression ratio of 20, JPEG performs equally well as the two top performing algorithms JPEG2000 and SPIHT, and even up to a compression ratio of 35 it is better as compared to PRVQ (which is up to 6 dB PSNR superior in this range !)

Overall, PSNR is found to be a less reliable indicator for recognition scores in the case of face images as compared to fingerprint images. This is somewhat surprising, since face images exhibit properties much more alike the common average image as compared to fingerprint images and one would expect a more reliable quality assessment by PSNR for this reason. In particular, the recognition performance of schemes involving JPEG is severely underestimated when using JPEGs PSNR performance as a reference. Similar to the fingerprint case, fractal compression turns out to be the least suited compression algorithm to be used within face recognition 
systems, a fact that would not have been expected based on PSNR measurements only. JPEG2000 and SPIHT are correctly predicted to deliver excellent matching robustness based on their superior PSNR values.

\section{CONCLUSIONS AND FUTURE WORK}

We have found PSNR to be a good indicator of finger and face recognition matching scores in the case of JPEG2000 and SPIHT. Both wavelet-based algorithms perform exceptionally well in terms of rate-distortion performance and matching scores of all recognition systems considered. While PSNR exactly predicts the poor matching scores of fractal compression the case of fingerprint images, the relatively high PSNR results for face images suggest fractal compression to perform superior to JPEG for this biometric modality. The opposite is true - despite the low PSNR results, JPEG performs quite well in face recognition applications for high and medium bitrate applications with respect to matching results. Given our findings, the use of JPEG and fractal compression at low bitrates may affect FRR/FNMR as well as even FAR/FMR in extreme cases for some recognition systems. These two algorithms should therefore not be used in such scenarios without checking possible side-effects.

In future we will cover two issues. First, we will investigate whether the good PSNR performance of some techniques tailored specifically to a target modality does carry over to recognition results. This will allow to answer the question if it makes sense to push the development of such specialized compression algorithms or if it is sufficient to stick to general purpose standards like JPEG2000. Second, we will model more accurately what really happens in a recognition system. Instead of matching a compressed and a raw version of a single fingerprint image, we will extend our approach by using the more or less compressed versions of fingerprint images corresponding to different acquisitions of a single finger in the matching stage.

\section{Acknowledgements}

The work described in this paper is partially supported by the Austrian Science Fund, project no. 15170.

\section{REFERENCES}

1. W. Pennebaker and J. Mitchell, JPEG - Still image compression standard, Van Nostrand Reinhold, New York, 1993.

2. J. N. Bradley, C. M. Brislawn, and T. Hopper, "The FBI wavelet/scalar quantization standard for gray-scale fingerprint image compression," in SPIE Proceedings, Visual Information Processing II, 1961, pp. 293-304, (Orlando, FL, USA), Apr. 1993.

3. D. Taubman and M. Marcellin, JPEG2000 - Image Compression Fundamentals, Standards and Practice, Kluwer Academic Publishers, 2002.

4. M. A. Lepley, "Profile for 1000ppi fingerprint compression," Tech. Rep. MTR 04B0000022, The MITRE Corporation, 2004.

5. Y. Fisher, T. Shen, and D. Rogovin, "A comparison of fractal methods with DCT (JPEG) and wavelets (EPIC)," in Neural and Stochastic Methods in Image and Signal Processing III, SPIE Proceedings 2304-16, (San Diego, CA, USA), July 1994.

6. D. Santa-Cruz and T. Ebrahimi, "A study of JPEG 2000 still image coding versus other standards," in Proceedings of the 10th European Signal Processing Conference, EUSIPCO '00, (Tampere, Finland), Sept. 2000.

7. A. Zhang, B. Cheng, R. Acharya, and R. Menon, "Comparison of wavelet transforms and fractal coding in texture-based image retrieval," in Visual Data Exploration and Analysis III, G. Grinstein and R. Erbacher, eds., SPIE Proceedings 2656, pp. 116-125, SPIE, Mar. 1996.

8. D. Krämer, A. Bruckmann, T. Freina, M. Reichl, and A. Uhl, "Comparison of wavelet, fractal and DCT based methods on the compression of prediction-error images," in Proceedings of the International Picture Coding Symposium (PCS'97), ITG-Fachberichte 143, pp. 393-397, VDE-Verlag, Berlin, Offenbach, Sept. 1997.

9. B. Jerabek, P. Schneider, and A. Uhl, "Comparison of lossy image compression methods applied to photorealistic and graphical images using public domain sources," Tech. Rep. RIST++15/98, Research Institute for Softwaretechnology, University of Salzburg, 1998. 
10. R. Kidd, "Comparison of wavelet scalar quantization and JPEG for fingerprint image compression," Journal of Electronic Imaging 4(1), pp. 31-39, 1995.

11. B. G. Sherlock and D. M. Monro, "Optimized wavelets for fingerprint compression," in Proceedings of the 1996 International Conference on Acoustics, Speech and Signal Processing (ICASSP'96), (Atlanta, GA, USA), May 1996.

12. B. G. Sherlock and D. M. Monro, "Psychovisually tuned wavelet fingerprint compression," in Proceedings of the IEEE International Conference on Image Processing (ICIP'96), IEEE Signal Processing Society, (Lausanne, Switzerland), Sept. 1996.

13. T.-K. Lin, "Wavelet compression of fingerprints and recognition using moment invariants," in IASTED Conference on Artificial Intelligence and Applications, (Innbruck, Austria), Feb. 2006. paper no. 502-031.

14. O. Gerek and A. Cetin, "Polyphase adaptive filter banks for fingerprint image compression," Electronics Letters 34, pp. 1931-1932, Oct. 1998.

15. B. G. Sherlock and D. M. Monro, "Balanced uncertainty wavelets for fingerprint compression," in IEE Colloquium on Image Processing for Security Applications, pp. 5-8, (London, GB), Mar. 1997.

16. U. Grasemann and R. Miikkulainen, "Effective image compression using evolved wavelets," in GECCO '05: Proceedings of the 2005 conference on Genetic and evolutionary computation, pp. 1961-1968, ACM Press, (New York, NY, USA), 2005.

17. J. Scharinger, H. Gaderbauer, and G. Wilflingseder, "Redesigning the FBI fingerprint compression standard," in Pattern Recognition and Medical Computer Vision 1998, M. Gengler, M. Prinz, and E. Schuster, eds., books@ocg.at 106, pp. 215-223, Austrian Computer Society, 1998.

18. S. Kasaei, M. Deriche, and B. Boashash, "A novel fingerprint image compression technique using wavelet packets and pyramid lattice vector quantization," IEEE Transactions on Image Processing 12(11), pp. 13651378, 2002.

19. P. Saeedian and B. Shirazi, "A novel fingerprint image compression technique using adaptive subband image coding," in Proceedings of the 2004 Picture Coding Symposium PCS'04, 2004.

20. G. Khuwaja, "Best parameter based compression of fingerprints with wavelet packets," International Journal of Computer Applications in Technology 19(1), pp. 51-62, 2004.

21. J. Thärna, K. Nilsson, and J. Bigun, "Orientation scanning to improve lossless compression of fingerprint images," in Proceedings of AVBPA, J. Kittler and M. Nixon, eds., LNCS 2688, pp. 343-350, Springer Verlag, 2003.

22. R. Sudhakar, N. Vigesh, and S. Jayaraman, "Application of wavelet footprints for fingerprint compression," ICGST International Journal on Graphics, Vision, and Image Processing 5(6), pp. 39-45, 2005.

23. A. Said and W. A. Pearlman, "A new, fast, and efficient image codec based on set partitioning in hierarchical trees," IEEE Transactions on Circuits and Systems for Video Technology 6, pp. 243-249, June 1996.

24. R. Sudhakar, R. Karthiga, and S. Jayaraman, "Fingerprint compression using contourlet transform with modified SPIHT algorithm," IJECE Iranian Journal of Electrical and Computer Engineering 5(1), pp. 3-10, 2005.

25. R. Eslami and H. Radha, "Wavelet-based contourlet transform and its application to image coding," in Proceedings of the IEEE International Conference on Image Processing (ICIP'04), IEEE Signal Processing Society, (Singapore), Oct. 2004.

26. R. Eslami and H. Radha, "Wavelet-based contourlet packet image coding," in 2005 Conference on Information Sciences and Systems, (The John Hopkins University), Mar. 2005.

27. C. Beleznai, H. Ramoser, B. Wachmann, J. Birchbauer, H. Bischof, and W. Kropatsch, "Memory-efficient fingerprint verification," in Proceedings of the IEEE International Conference on Image Processing (ICIP'01), 2, pp. 463-466, (Thessaloniki, Greece), Oct. 2001.

28. X. Li, J.-H. Lai, and Z. Zhang, "An efficient compression and reconstruction method of face image for low rate net," in Advances in Biometric Person Authentication, 5th Chinese Conference on Biometric Recognition, SINOBIOMETRICS 2004, LNCS 3338, pp. 379-385, Springer-Verlag, 2004.

29. K. Delac, M. Grigic, and S. Grigic, "Effects of JPEG and JPEG2000 compression on face recognition," in Proceedings of ICAPR 2005, LNCS 3687, pp. 136-145, Springer-Verlag, 2005. 
30. D. McGarry, C. Arndt, S. McCabe, and D. D'Amato, "Effects of compression and individual variability on face recognition performance," in Biometric Technology for Human Identification, Proceedings of SPIE 5404, pp. 362-372, Aug. 2004.

31. Y. Fisher, ed., Fractal Image Compression: Theory and Application, Springer-Verlag, New York, 1995. 